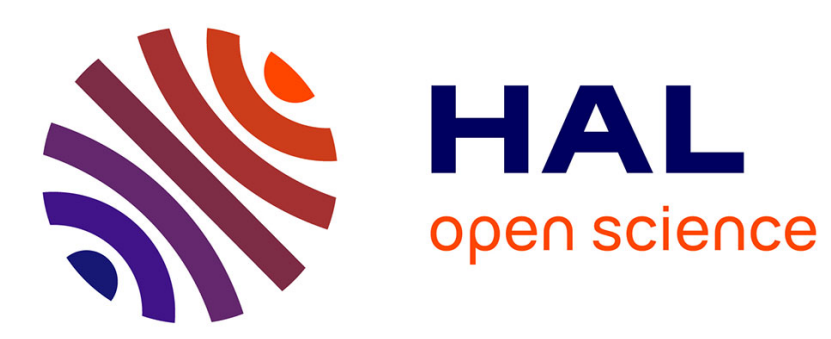

\title{
Internal exponential stabilization to a nonstationary solution for 1D Burgers equations with piecewise constant controls
}

Axel Kröner, Sergio S. Rodrigues

\section{- To cite this version:}

Axel Kröner, Sergio S. Rodrigues. Internal exponential stabilization to a nonstationary solution for 1D Burgers equations with piecewise constant controls. Control Conference (ECC), 2015 European, Jul 2015, Linz, Austria. pp.2676-2681, 10.1109/ECC.2015.7330942 . hal-01089896

\section{HAL Id: hal-01089896 https://hal.science/hal-01089896}

Submitted on 2 Dec 2015

HAL is a multi-disciplinary open access archive for the deposit and dissemination of scientific research documents, whether they are published or not. The documents may come from teaching and research institutions in France or abroad, or from public or private research centers.
L'archive ouverte pluridisciplinaire HAL, est destinée au dépôt et à la diffusion de documents scientifiques de niveau recherche, publiés ou non, émanant des établissements d'enseignement et de recherche français ou étrangers, des laboratoires publics ou privés. 


\title{
Internal exponential stabilization to a nonstationary solution for 1D Burgers equations with piecewise constant controls
}

\author{
Axel Kröner and Sérgio S. Rodrigues
}

\begin{abstract}
The feedback stabilization of the Burgers system to a nonstationary solution using a finite number of internal piecewise constant controls is considered. Estimates for the number of needed controls are derived. In the particular case of no constraint on the support of the control a better estimate is derived, so the possibility of getting an analogous estimate for the general case is discussed. That possibility is suggested by the results of some numerical simulations.
\end{abstract}

\section{INTRODUCTION}

Let $L>0$ be a positive real number. We consider the controlled Burgers equations in the interval $\Omega=(0, L) \subset \mathbb{R}$ :

$$
\partial_{t} u+u \partial_{x} u-\nu \partial_{x x} u+h+\zeta=0,\left.\quad u\right|_{\Gamma}=0 .
$$

Here $u$ stands for the unknown velocity of the fluid, $\nu>0$ is the viscosity, $h$ is a fixed function, $\Gamma=\partial \Omega$ stands for the boundary $\{0, L\}$ of $\Omega$, and $\zeta$ is a control taking values in the space of square-integrable functions in $\Omega$, whose support, in $x$, is contained in a given open subset $\mathcal{O} \subset \Omega$. The Burgers equation models gas dynamics and traffic flow.

Let us be given a positive constant $\lambda>0$, a continuous Lipschitz function $\chi \in W^{1, \infty}(\Omega, \mathbb{R})$ with nonempty support $\emptyset \neq \operatorname{supp}(\chi) \subseteq \overline{\mathcal{O}} \subseteq \bar{\Omega}$, and a time-dependent solution $\hat{u} \in$ $\mathcal{W}$ of (1), with $\zeta=0$, in a suitable Banach space $\mathcal{W}$. Let $\mathcal{O}=\left(l_{1}, l_{2}\right) \subseteq(0, L)$; in [11] it is shown the existence of an integer $M$ and a function $\eta=\eta(t, x)$, defined for $t>0, x \in$ $\Omega$, such that the solution $u=u(t, x)$ of problem (1), with $\zeta=\chi P_{M}\left(\left.\eta\right|_{\mathcal{O}}\right)$, and supplemented with the initial condition

$$
u(0, x)=u_{0}(x)
$$

is defined on $[0,+\infty)$ and satisfies the relation $\mid u(t)-$ $\left.\hat{u}(t)\right|_{L^{2}(\Omega, \mathbb{R})} ^{2} \leq C \mathrm{e}^{-\lambda t}|u(0)-\hat{u}(0)|_{L^{2}(\Omega, \mathbb{R})}^{2}$, provided $\mid u(0)-$ $\left.\hat{u}(0)\right|_{L^{2}(\Omega, \mathbb{R})}<\epsilon$, for small enough $\epsilon$. Here $M, C$, and $\epsilon$ can be taken depending only on $\left(|\hat{u}|_{\mathcal{W}}, \lambda\right)$, and $P_{M}$ is the orthogonal projection in $L^{2}(\mathcal{O}, \mathbb{R})$ onto the subspace $L_{M}^{2}(\mathcal{O}, \mathbb{R}):=\operatorname{span}\left\{\sin \left(\frac{i \pi\left(x-l_{1}\right)}{l_{2}-l_{1}}\right) \mid i \in \mathbb{N}, 1 \leq i \leq M\right\}$. That is, the internal control $\zeta=\chi P_{M}\left(\left.\eta\right|_{\mathcal{O}}\right)$ stabilizes exponentially, with rate $\frac{\lambda}{2}$, the Burgers system to the targeted nonstationary reference trajectory $\hat{u}=\hat{u}(t)$.

In the case we take $\chi(x)=1$ for all $x \in \Omega$, then it is shown in [11, Section 3] that it is enough to take

$$
M \geq D_{0}\left(\nu^{-2}|\hat{u}|_{\mathcal{W}}^{2}+\nu^{-1} \lambda\right)^{\frac{1}{2}},
$$

A. K. is with INRIA Saclay and CMAP, Ecole Polytechnique, Route de Saclay, 91128 Palaiseau cedex, France. axel.kroenerdinria.fr

S. R. is with Johann Radon Institute for Computational and Applied Mathematics (RICAM), ÖAW, 4040 Linz, Austria, and aknowledges partial support from the Austrian Science Fund (FWF): P 26034-N25. sergio.rodrigues@oeaw.ac.at while in the general case our control is supported in a small subset $\operatorname{supp}(\chi) \subseteq \mathcal{O}$, it is shown that it is "enough" to take

$$
M \geq D_{1} \mathrm{e}^{D_{2}\left(1+\left(\nu^{-1} \lambda\right)^{\frac{1}{2}}+\left(\nu^{-1} \lambda\right)^{\frac{2}{3}}+\nu^{-1}|\hat{u}|_{\mathcal{W}}+\nu^{-2}|\hat{u}|_{\mathcal{W}}^{2}\right)}
$$

where $D_{0}, D_{1}$, and $D_{2}$ are constants depending on $\chi$ and $\Omega$. Since the estimates are of different nature some numerical simulations have been presented in [11] suggesting that an estimate like (3) should also hold in the general case of localized controls.

In the derivation of the estimate (3) it is used explicitly the fact that the controls $\sin \left(\frac{i \pi\left(x-l_{1}\right)}{l_{2}-l_{1}}\right), 1 \leq i \leq M$, are eigenfunctions of the Dirichlet Laplace operator in $L^{2}(\mathcal{O}, \mathbb{R})$. In applications the type of controls at our disposal may be not of this type, so one question arises: can we derive similar estimates for other type of controls? Here we show that this is the case for piecewise constant controls; more precisely if we consider a partition $l_{1}=p_{0}<p_{1}<p_{2}<\cdots<$ $p_{M-1}<p_{M}=l_{2}$ of $\mathcal{O}$ and at each instant of time we apply a control that vanishes outside $\mathcal{O}$ and takes a constant value $\zeta_{i}(t, x)=\zeta_{i}(t)$ for all $x \in\left(p_{i-1}, p_{i}\right), i \in\{1,2, \ldots, M\}$. Notice that in this case we have $M$ independent piecewise constant controls at our disposal.

In the special case the partition is uniform we find estimates analogous to (3) and (4) for the cases the support of the control is either the entire $\Omega$ or a strict subset of $\Omega$, respectively. In the case of a not necessarily uniform partition we find a sufficient condition for stabilization that is given in terms of the maximum $\max \left\{p_{i-1}-p_{i} \mid 1 \leq i \leq M\right\}$.

We present also the results of some of the numerical simulations we have performed, that suggest that the possibility of getting, also in the general case, an estimate analogous to (3) is plausible.

We underline that our targeted trajectory $\hat{u}(t)$ is nonstationary, and that in this case there are only a few works in the mathematical literature concerning the feedback stabilization to $\hat{u}$ and the estimation on the dimension of the controller; we refer to [4], [11]. This case is important when time dependent external forces $h=h(t)$ act on the system (1). Since these problems arise in applications, methods to solve these problems numerically have already been developed (see, e.g., [7], [9], [10] and references therein). We refer also the reader to [12] (in particular, see Section 4 concerning trajectory tracking) and to [8] (in particular, see Section 7.1 concerning linear feedback control of Navier-Stokes flows).

Notice that, as mentioned in [4], [11], [14] the nonstationarity of the trajectory requires different tools than those used in the stationary case, which has been studied along the last 
decade; see for example [1]-[3], [5], [13].

The rest of the paper is organized as follows. In Section II we recall some well-known results and set up our problem. In Section III, for the linear Oseen-Burgers system, we present a general sufficient condition for stabilization that the family of controls has to satisfy; Section III-A deals with the particular case where we impose no restriction on the support of the control and Section III-B deals with the general case. In Section IV we consider the case of piecewise constant controls where the sufficient condition is given in terms of the maximum length among the subintervals of the partition of the control region. In Section V we give the local result for the Burgers system. In Sections VI and VII we present the results of some simulations we have performed for the Oseen-Burgers system and Burgers system, respectively.

Notation. We write $\mathbb{R}$ and $\mathbb{N}$ for the sets of real numbers and nonnegative integers, respectively, and we define $\mathbb{R}_{r}:=$ $(r,+\infty)$, for $r \in \mathbb{R}$, and $\mathbb{N}_{0}:=\mathbb{N} \backslash\{0\}$. Given an open interval $I \subseteq \mathbb{R}$ and two Banach spaces $X$ and $Y$, we write $W(I, X, Y):=\left\{f \in L^{2}(I, X) \mid \partial_{t} f \in L^{2}(I, Y)\right\}$, where the derivative $\partial_{t} f$ is taken in the sense of distributions. This space is endowed with the natural norm $|f|_{W(I, X, Y)}:=$ $\left(|f|_{L^{2}(I, X)}^{2}+\left|\partial_{t} f\right|_{L^{2}(I, Y)}^{2}\right)^{\frac{1}{2}}$. In the case $X=Y$ we write $H^{1}(I, X):=W(I, X, X)$. The space of continuous linear mappings from $X$ into $Y$ will be denoted by $\mathcal{L}(X \rightarrow Y)$.

Also, $C, C_{i}, i \in \mathbb{N}$, stand for generic positive constants.

\section{Preliminaries}

Let $\Omega=(0, L)$, with $L>0$. We will denote $V:=$ $H_{0}^{1}(\Omega, \mathbb{R}), H:=L^{2}(\Omega, \mathbb{R}), \mathrm{D}\left(\partial_{x x}\right):=V \cap H^{2}(\Omega, \mathbb{R})$, and $V^{\prime}:=H^{-1}(\Omega, \mathbb{R})$. As usual the space $H$ is taken as a pivot space, $H=H^{\prime}$, and $V^{\prime}$ is the dual of $V$. We also denote

$$
\mathcal{W}:=L^{\infty}\left(\mathbb{R}_{0}, L^{\infty}(\Omega, \mathbb{R})\right)
$$

and, for given Banach spaces $X$ and $Y$,

$$
\begin{aligned}
L_{\text {loc }}^{2}\left(\mathbb{R}_{0}, X\right) & :=\left\{f|f|_{(0, T)} \in L^{2}((0, T), X), T>0\right\}, \\
W_{\text {loc }}\left(\mathbb{R}_{0}, X, Y\right) & :=\left\{f|f|_{(0, T)} \in W((0, T), X, Y), T>0\right\} .
\end{aligned}
$$

Fix a function $h \in L_{\text {loc }}^{2}\left(\mathbb{R}_{0}, V^{\prime}\right)$ and suppose that $\hat{u} \in$ $\mathcal{W} \cap W_{\text {loc }}\left(\mathbb{R}_{0}, V, V^{\prime}\right)$ solves the Burgers system (1), with $\zeta=0$ and initial condition $\hat{u}_{0}:=\hat{u}(0) \in H$.

Let us be given $\lambda>0$, a function $u_{0}$ such that $\left|u_{0}-\hat{u}(0)\right|_{H}$ is small enough, and an open interval $\mathcal{O}=\left(l_{1}, l_{2}\right) \subseteq \Omega$.

Let $\mathcal{C}=\left\{\Psi_{i} \in L^{2}(\Omega, \mathbb{R}) \mid i \in\{1,2, \ldots, M\}\right\}$ be a family of controls such that $\operatorname{supp}\left(\Psi_{i}\right) \subseteq \overline{\mathcal{O}}$. Let us denote by $P_{M}$ the orthogonal projection in $L^{2}(\Omega, \mathbb{R})$ onto the linear space $\mathcal{S}_{\mathcal{C}}:=\operatorname{span} \mathcal{C}$ spanned by the functions in $\mathcal{C}$.

Our goal is to find a control $\eta \in L^{2}\left(\mathbb{R}_{0}, H\right)$ such that the solution of the problem (1)-(2), with $\zeta=P_{M} \eta$ is defined for all $t>0$ and converges exponentially to $\hat{u}$, that is, for some positive constant $C>0$ independent of $u_{0}-\hat{u}_{0}$,

$$
|u(t)-\hat{u}(t)|_{H}^{2} \leq C \mathrm{e}^{-\lambda t}\left|u_{0}-\hat{u}_{0}\right|_{H}^{2} \quad \text { for } t \geq 0 .
$$

Seeking for the control $\eta$ and considering the corresponding solution $u$, we find that $v=u-\hat{u}$, will solve the nonlinear
Oseen-Burgers-like system

$$
\partial_{t} v-\nu \partial_{x x} v+v \partial_{x} v+\partial_{x}(\hat{u} v)+\zeta=0, \quad v(0)=v_{0},
$$

with $\left.v\right|_{\Gamma}=0, \zeta=P_{M} \eta$ and $v_{0}=u(0)-\hat{u}(0)$. It is now clear that to achieve (6) it suffices to consider the problem of local exponential stabilization to zero for solutions of (7), where "local" means that the property is to hold "provided $\left|v_{0}\right|_{H}$ is small enough".

The existence and uniqueness of a weak solution $v \in$ $W_{\text {loc }}\left(\mathbb{R}_{0}, V, V^{\prime}\right)$ for system (7) can be proved by classical arguments (cf. [11, Section 2.2]).

\section{The OSEen-Burgers SYSTEM.}

Following [11, Section 3] we start by looking for a control in the form $\zeta=P_{M} \eta$, with $\eta \in L^{2}\left(\mathbb{R}_{0}, H\right)$, that stabilizes exponentially the linear Oseen-Burgers system

$$
\partial_{t} v-\nu \partial_{x x} v+\partial_{x}(\hat{u} v)+\zeta=0,\left.\quad v\right|_{\Gamma}=0, \quad v(0)=v_{0},
$$

to zero, with a desired exponential rate $\frac{\lambda}{2}>0$. System (8) is well-posed (cf. [11]). Recall that $P_{M}$ is the orthogonal projection onto $\mathcal{S}_{\mathcal{C}}=\operatorname{span} \mathcal{C}$ and $\mathcal{C}=\left\{\Psi_{i} \in L^{2}(\Omega, \mathbb{R}) \mid i \in\right.$ $\{1,2, \ldots, M\}\}$; so the question is which conditions must the family $\mathcal{C}$ satisfy in order to guarantee the existence of such a function $\eta$.

The results will follow for system (7), provided $\left|v_{0}\right|_{H}$ is small enough, by a fixed point argument.

\section{A. The particular case $\mathcal{O}=\Omega$.}

In the case of $\mathcal{O}=\Omega$ we have no constraint in the support of the controller.

Theorem 3.1: For given $\hat{u} \in \mathcal{W}$ and $\lambda>0$, suppose that

$$
\left|\left(1-P_{M}\right)\right|_{\mathcal{L}\left(H \rightarrow V^{\prime}\right)}^{2} \leq \frac{2}{3 \mathrm{e}^{1}}\left(\nu^{-2}|\hat{u}|_{\mathcal{W}}^{2}+\nu^{-1} \lambda\right)^{-1},
$$

where e is the Napier's constant. Then for any given $v_{0} \in$ $H$, there is a control $\eta^{\lambda, \hat{u}, \nu}\left(v_{0}\right) \in L^{2}\left(\mathbb{R}_{0}, H\right)$ such that the corresponding solution $v$ of system (8), with $\zeta=$ $\chi \mathbb{E}_{0}^{\mathcal{O}} P_{M}^{\mathcal{O}}\left(\left.\eta^{\lambda, \hat{u}, \nu}\right|_{\mathcal{O}}\right)$, satisfies the inequality

$$
|v(t)|_{H}^{2} \leq 2\left(1+\mathrm{e}^{\frac{1}{2}}\right) \mathrm{e}^{-\lambda t}\left|v_{0}\right|_{H}^{2}, \quad t \geq 0 .
$$

Moreover, the mapping $v_{0} \mapsto \eta^{\lambda, \hat{u}, \nu}\left(v_{0}\right)$ is well defined, is linear, and satisfies for $0 \leq \hat{\lambda}<\lambda$, the estimate

$$
\begin{aligned}
& \left|\mathrm{e}^{(\hat{\lambda} / 2) t} \eta^{\lambda, \hat{u}, \nu}\left(v_{0}\right)\right|_{L^{2}\left(\mathbb{R}_{0}, H\right)}^{2} \leq \frac{4 \mathrm{e}^{\frac{1}{2}}}{\lambda-\hat{\lambda}}\left(\frac{1}{\nu}|\hat{u}|_{\mathcal{W}}^{2}+\lambda\right)\left|v_{0}\right|_{H}^{2} . \\
& \quad \text { Proof: Let } w \text { solve }
\end{aligned}
$$

$$
\partial_{t} w=\nu \partial_{x x} w-\partial_{x}(\hat{u} w)+\frac{\lambda}{2} w,\left.\quad w\right|_{\Gamma}=0, \quad w(0)=v_{0} .
$$

By standard arguments, following [11, Section 3.1], we find

$$
\begin{aligned}
\frac{\mathrm{d}}{\mathrm{d} t}|w|_{H}^{2} & \leq \frac{1}{2 \nu}|\hat{u}|_{L^{\infty}(\Omega, \mathbb{R})}^{2}|w|_{H}^{2}+\lambda|w|_{H}^{2} ; \\
|w|_{L^{\infty}((0, T), H)}^{2} & \leq \mathrm{e}^{\left(\frac{1}{2 \nu}|\hat{u}|_{\mathcal{W}}^{2}+\lambda\right) T}\left|v_{0}\right|_{H}^{2} .
\end{aligned}
$$

Setting $\varphi(t):=1-\frac{t}{T} \in C^{1}([0, T], \mathbb{R}), \delta:=\varphi w$ solves

$$
\partial_{t} \delta=\nu \partial_{x x} \delta-\partial_{x}(\hat{u} \delta)+\frac{\lambda}{2} \delta-\frac{1}{T} w,\left.\quad \delta\right|_{\Gamma}=0, \quad \delta(0)=v_{0}
$$


with $\delta(T)=0$. Let now $\delta_{M}$ be the solution of the system

$$
\begin{aligned}
& \partial_{t} \delta_{M}=\nu \partial_{x x} \delta_{M}-\partial_{x}\left(\hat{u} \delta_{M}\right)+\frac{\lambda}{2} \delta_{M}-\frac{1}{T} P_{M} w, \\
& \left.\delta_{M}\right|_{\Gamma}=0, \quad \delta_{M}(0)=v_{0} .
\end{aligned}
$$

The difference $d:=\delta-\delta_{M}$ solves

$$
\begin{aligned}
& \partial_{t} d=\nu \partial_{x x} d-\partial_{x}(\hat{u} d)+\frac{\lambda}{2} d-\frac{1}{T}\left(1-P_{M}^{\Omega}\right) w, \\
& \left.d\right|_{\Gamma}=0, \quad d(0)=0 .
\end{aligned}
$$

Then, proceeding as in [11, Section 3.1] we can arrive to $|d|_{L^{\infty}\left(\left(0, T_{*}\right), H\right)}^{2} \leq \frac{3 \mathrm{e}^{1}}{2 \nu}\left(\nu^{-1}|\hat{u}|_{\mathcal{W}}^{2}+\lambda\right)\left|\left(1-P_{M}\right)\right|_{\mathcal{L}\left(H \rightarrow V^{\prime}\right)}^{2}\left|v_{0}\right|_{H}^{2}$ with $T_{*}:=\frac{1}{2\left(\nu^{-1}|\hat{u}|^{2}+\lambda\right)}$. Now, recalling that $\delta_{M}(0)=v_{0}$ and $\delta_{M}\left(T_{*}\right)=-d\left(T_{*}\right)$, we find that if (9) holds then $\left|\delta_{M}\left(T_{*}\right)\right|_{H}^{2} \leq\left|\delta_{M}(0)\right|_{H}^{2}$.

Furthermore, $\left|\delta_{M}\right|_{L^{\infty}\left(\left(0, T_{*}\right), H\right)}^{2}=|\delta-d|_{L^{\infty}\left(\left(0, T_{*}\right), H\right)}^{2} \leq$ $2|\varphi w|_{L^{\infty}\left(\left(0, T_{*}\right), H\right)}^{2}+2|d|_{L^{\infty}\left(\left(0, T_{*}\right), H\right)}^{2^{*}} \leq C_{M}^{\delta}\left|\delta_{M}(0)\right|_{H}^{2}$, with

$$
C_{M}^{\delta}:=2 \mathrm{e}^{\left(\frac{1}{2 \nu}|\hat{u}|_{\mathcal{W}}^{2}+\lambda\right) T^{*}}+2 \leq 2\left(\mathrm{e}^{\frac{1}{2}}+1\right)=: \Upsilon_{\delta}
$$

Next, we may consider the system (11) in $\left(T_{*},+\infty\right) \times \Omega$ with $w\left(T_{*}\right)=\delta_{M}\left(T_{*}\right)$, and repeat the arguments. Recursively, we conclude that in each interval $J_{*}^{i}:=\left(i T_{*},(i+\right.$ 1) $\left.T_{*}\right), i \in \mathbb{N}_{0}$, we have $\left|\delta_{M}\left((i+1) T_{*}\right)\right|_{H}^{2} \leq\left|\delta_{M}\left(i T_{*}\right)\right|_{H}^{2}$ and $\left|\delta_{M}\right|_{L^{\infty}\left(J_{*}^{i}, H\right)}^{2} \leq \Upsilon_{\delta}\left|\delta_{M}\left(i T_{*}\right)\right|_{H}^{2}$. Hence, the concatenated solution satisfy $\left|\delta_{M}\right|_{L^{\infty}\left(\mathbb{R}_{0}, H\right)}^{2} \leq \Upsilon_{\delta}\left|v_{0}\right|_{H}^{2}$.

Next we notice that $v:=\mathrm{e}^{-\frac{\lambda}{2} t} \delta_{M}$ solves (8), in $\mathbb{R}_{0} \times \Omega$, with the concatenated control $\zeta=P_{M}\left(\mathrm{e}^{-\frac{\lambda}{2} t}\left(-T_{*}^{-1}\right) w\right)=$ $-T_{*}^{-1} \mathrm{e}^{-\frac{\lambda}{2} t} P_{M} w$, where $\left.w\right|_{J^{i}}=: w_{i}$ solves $(11)$, in $J_{*}^{i} \times \Omega$, with $w_{i}\left(i T_{*}\right)=w\left(i T_{*}\right)=\delta_{M}^{*}\left(i T_{*}\right)$; from the boundedness of $\left\{\left|\delta_{M}\left(i T_{*}\right)\right|_{H} \mid i \in \mathbb{N}\right\}$, we can conclude that the family $\left\{|w|_{L^{2}\left(J_{*}^{i}, H\right)} \mid i \in \mathbb{N}\right\}$ is bounded; so we have that $\mathrm{e}^{\frac{\hat{\lambda}}{2} t} \zeta \in$ $L^{2}\left(\mathbb{R}_{0}, H\right)$ for all $\hat{\lambda}<\lambda$. Finally we observe that $|v(t)|_{H}^{2} \leq$ $\mathrm{e}^{-\lambda t}\left|\delta_{M}\right|_{L^{\infty}\left(\mathbb{R}_{0}, H\right)}^{2} \leq \Upsilon_{\delta} \mathrm{e}^{-\lambda t}\left|v_{0}\right|_{H}^{2}$, and that for $\eta^{\lambda, \hat{u}, \nu}:=$ $\mathrm{e}^{-\frac{\lambda}{2} t}\left(-T_{*}^{-1}\right) w$ we have

$$
\begin{aligned}
& \left|\mathrm{e}^{\frac{\hat{\lambda}}{2} t} \eta^{\lambda, \hat{u}, \nu}\right|_{L^{2}\left(\mathbb{R}_{0}, H\right)}^{2}=\int_{\mathbb{R}_{0}} \mathrm{e}^{(\hat{\lambda}-\lambda) s} T_{*}^{-2}|w(s)|_{H}^{2} \mathrm{~d} s \\
\leq & \frac{1}{\lambda-\hat{\lambda}} T_{*}^{-2} \mathrm{e}^{\left(\frac{1}{2 \nu}|\hat{u}|_{\mathcal{W}}^{2}+\lambda\right) T^{*}}\left|v_{0}\right|_{H}^{2} \\
\leq & \frac{1}{\lambda-\hat{\lambda}}\left(2\left(\nu^{-1}|\hat{u}|_{\mathcal{W}}^{2}+\lambda\right)\right)^{2} \mathrm{e}^{\frac{1}{2}}\left|v_{0}\right|_{H}^{2} .
\end{aligned}
$$

That is, $\left|\mathrm{e}^{\frac{\hat{\lambda}}{2} t} \eta^{\lambda, \hat{u}, \nu}\right|_{L^{2}\left(\mathbb{R}_{0}, H\right)}^{2} \leq \frac{4 \mathrm{e}^{\frac{1}{2}}}{\lambda-\hat{\lambda}}\left(\nu^{-1}|\hat{u}|_{\mathcal{W}}^{2}+\lambda\right)^{2}\left|v_{0}\right|_{H}^{2}$, which ends the proof.

\section{B. The general case}

In the case $\mathcal{O} \neq \Omega$ we have the following result, whose proof follows by similar arguments as in [11, Section 3.2] and in the previous Section III-A; thus we skip the details.

Theorem 3.2: Let us be given $\hat{u} \in \mathcal{W}$ and $\lambda>0$. Then there are constants $D_{1}$ and $D_{2}$ depending only in $\mathcal{O}$ and $\Omega$ with the following property: if

$$
\begin{aligned}
& \left|\left(1-P_{M}\right)\right|_{\mathcal{L}\left(H \rightarrow V^{\prime}\right)}^{2} \\
\leq & D_{1} \mathrm{e}^{-D_{2}\left(1+\left(\frac{\lambda}{\nu}\right)^{\frac{1}{2}}+\left(\frac{\lambda}{\nu}\right)^{\frac{2}{3}}+\frac{|\hat{u}|_{\mathcal{W}}}{\nu}+\frac{|\hat{u}|_{\mathcal{W}}^{2}}{\nu^{2}}\right)},
\end{aligned}
$$

then for any given $v_{0} \in H$, there is a control $\eta^{\lambda, \hat{u}, \nu}\left(v_{0}\right) \in$ $L^{2}\left(\mathbb{R}_{0}, H\right)$ such that, taking $\zeta=P_{M} \eta^{\lambda, \hat{u}, \nu}$, the corresponding solution $v$ of system (8) satisfies, for $t \geq 0$, the inequality

$$
|v(t)|_{H}^{2} \leq K_{\chi, \Omega} \mathrm{e}^{-\lambda t}\left|v_{0}\right|_{H}^{2}
$$

with $K_{\chi, \Omega}$ depending on $D_{1}, D_{2}, \frac{\lambda}{\nu}$, and $\frac{|\hat{u}|_{\mathcal{W}}}{\nu}$. Moreover, the mapping $v_{0} \mapsto \eta^{\lambda, \hat{u}, \nu}\left(v_{0}\right)$ is well defined, is linear, and satisfies for $0 \leq \hat{\lambda}<\lambda$ the inequality

$$
\begin{aligned}
& \left|\mathrm{e}^{\frac{\hat{\lambda}}{2} t} \eta^{\lambda, \hat{u}, \nu}\left(v_{0}\right)\right|_{L^{2}\left(\mathbb{R}_{0}, H\right)}^{2} \\
\leq & \frac{\nu \mathrm{e}^{D_{3}\left(1+3\left(\frac{\lambda}{\nu}\right)^{\frac{1}{2}}+\left(\frac{\lambda}{\nu}\right)^{\frac{2}{3}}+3 \frac{1}{\nu}|\hat{u}|_{\mathcal{W}}+\frac{1}{\nu^{2}}|\hat{u}|_{\mathcal{W}}^{2}\right)}}{1-\mathrm{e}^{(\hat{\lambda}-\lambda)\left(2\left(\nu \lambda+|\hat{u}|_{\mathcal{W}}^{2}\right)\right)^{-\frac{1}{2}}}}\left|v_{0}\right|_{H}^{2} .
\end{aligned}
$$

\section{The gap between (9) and (12).}

Comparing estimates (9) and (12), we see that there is a big gap; the former being proportional to $\left(\frac{1}{\nu^{2}}|\hat{u}|_{\mathcal{W}}^{2}+\right.$ $\left.\frac{1}{\nu} \lambda\right)^{\frac{1}{2}}$, while the latter depends exponentially on both $\frac{1}{\nu}|\hat{u}|_{\mathcal{W}}$ and $\left(\frac{\lambda}{\nu}\right)^{\frac{1}{2}}$. For application purposes the latter is much less convenient, so one question arises naturally: can we improve (12)?

It seems that the idea used to derive (9) cannot (at least not straightforwardly) be applied in the general case. On the other side to derive (12) we start from an exact null controllability result (from [6]) and carry the cost of the respective control. This means that to improve (12) we will probably need a different idea.

\section{EXAMPLE: PIECEWISE CONSTANT CONTROLS}

We consider a partition

$$
l_{1}=p_{0}<p_{1}<p_{2}<\cdots<p_{M-1}<p_{M}=l_{2}
$$

of the controlled region $\mathcal{O}=\left(l_{1}, l_{2}\right)$, and at each instant of time we apply a control that vanishes outside $\mathcal{O}$ and takes a constant value $\eta_{i}(t, x)=\eta_{i}(t)$ for all $x \in\left(p_{i-1}, p_{i}\right)$, $i \in\{1,2, \ldots, M\}$; we suppose we can act independently in each subinterval $\mathcal{O}_{i}:=\left(p_{i-1}, p_{i}\right)$. That is, we have $M$ independent piecewise constant controls at our disposal, namely the functions in $\mathcal{C}:=\left\{\Psi_{i}=\mathcal{I}_{i} 1_{\Omega} \mid 1 \leq i \leq M\right\}$, where $1_{\Omega} \in L^{2}(\Omega, \mathbb{R})$ is the constant function $1_{\Omega}(x)=1$ for all $x \in \Omega$. and $\mathcal{I}_{i}: H^{1}(\Omega, \mathbb{R}) \rightarrow L^{2}(\Omega, \mathbb{R})$, denotes the indicator operator, $\mathcal{I}_{i} f(x):= \begin{cases}f(x), & \text { if } x \in \mathcal{O}_{i} \\ 0, & \text { if } x \in \Omega \backslash \overline{\mathcal{O}_{i}} .\end{cases}$

\section{A. An estimate for the dimension $M$ of the controller}

Let $P_{M}$ be the orthogonal projection in $L^{2}(\Omega, \mathbb{R})$ onto $\mathcal{S}_{\mathcal{C}}=\operatorname{span} \mathcal{C}$. Let also $P_{M}^{i}: L^{2}(\mathcal{O}, \mathbb{R}) \rightarrow \mathbb{R} \Psi_{i}$ be the orthogonal projection onto the one dimensional space spanned by $\Psi_{i}$. Observe that $P_{M}=\sum_{i=1}^{M} P_{M}^{i}$ and that, for given $u \in L^{2}(\Omega, \mathbb{R})$ and $v \in V$ with $\left.u\right|_{\Omega \backslash \overline{\mathcal{O}}}=0$ and $|v|_{V}=1$, we have

$$
\begin{aligned}
& \left|\left\langle\left(1-P_{M}\right) u, v\right\rangle_{V^{\prime}, V}\right|_{\mathbb{R}}=\left|\left(u,\left(1-P_{M}\right) v\right)_{H}\right|_{\mathbb{R}} \\
\leq & \sum_{i=1}^{M}\left|\left(\left.u\right|_{\mathcal{O}_{i}},\left.\left(1-P_{M}^{i}\right) v\right|_{\mathcal{O}_{i}}\right)_{L^{2}\left(\mathcal{O}_{i}, \mathbb{R}\right)}\right|_{\mathbb{R}} \\
\leq & \left.\left.\sum_{i=1}^{M}|u|_{\mathcal{O}_{i}}\right|_{L^{2}\left(\mathcal{O}_{i}, \mathbb{R}\right)}\left|\left(\left(1-P_{M}^{i}\right) v\right)\right|_{\mathcal{O}_{i}}\right|_{L^{2}\left(\mathcal{O}_{i}, \mathbb{R}\right)} .
\end{aligned}
$$


Notice that $\left.\left(\left(1-P_{M}^{i}\right) v\right)\right|_{\mathcal{O}_{i}}=\left(1-P_{M}^{i}\right) \mathcal{I}_{i} v \in L^{2}\left(\mathcal{O}_{i}, \mathbb{R}\right)$ is zero averaged, which implies $\left.\left|\left(\left(1-P_{M}^{i}\right) v\right)\right|_{\mathcal{O}_{i}}\right|_{L^{2}\left(\mathcal{O}_{i}, \mathbb{R}\right)} \leq$ $\frac{\hat{l}_{i}}{\pi}\left|\partial_{x}\left(\left.\left(\left(1-P_{M}^{i}\right) \mathcal{I}_{i} v\right)\right|_{\mathcal{O}_{i}}\right)\right|_{L^{2}\left(\mathcal{O}_{i}, \mathbb{R}\right)}=\left.\frac{\hat{l}_{i}}{\pi}\left|\partial_{x} v\right|_{\mathcal{O}_{i}}\right|_{L^{2}\left(\mathcal{O}_{i}, \mathbb{R}\right)}$, where $\hat{l}_{i}:=p_{i}-p_{i-1}$ is the length of $\mathcal{O}_{i}$. In particular, $\left.\left|\left(\left(1-P_{M}^{i}\right) v\right)\right|_{\mathcal{O}_{i}}\right|_{L^{2}\left(\mathcal{O}_{i}, \mathbb{R}\right)} \leq \frac{\hat{l}_{i}}{\pi}|v|_{V}=\frac{\hat{l}_{i}}{\pi}$.

Therefore, setting $\hat{l}=\max \left\{\hat{l}_{i} \mid 1 \leq i \leq M\right\}$, from (14) it follows that $\left|\left\langle\left(1-P_{M}\right) u, v\right\rangle_{V^{\prime}, V}\right|_{\mathbb{R}} \leq \frac{\hat{l}}{\pi}|u|_{H}$, and $\left|\left(1-P_{M}\right)\right|_{\mathcal{L}\left(H \rightarrow V^{\prime}\right)} \leq \frac{\hat{l}}{\pi}$.

In the case $\mathcal{O}=\Omega$, from Theorem 3.1 it is enough to take a partition of $\Omega$ such that

$$
\hat{l} \leq \pi\left(\frac{2}{3 \mathrm{e}^{1}}\right)^{\frac{1}{2}}\left(\nu^{-2}|\hat{u}|_{\mathcal{W}}^{2}+\nu^{-1} \lambda\right)^{-\frac{1}{2}},
$$

which implies that $L \leq M \pi\left(\frac{2}{3 \mathrm{e}^{1}}\right)^{\frac{1}{2}}\left(\nu^{-2}|\hat{u}|_{\mathcal{W}}^{2}+\nu^{-1} \lambda\right)^{-\frac{1}{2}}$, because $L \leq M \hat{l}$. Thus we need to take at least $M$ controls with $M$ satisfying

$$
M \geq \frac{L}{\pi}\left(\frac{3 \mathrm{e}^{1}}{2}\right)^{\frac{1}{2}}\left(\nu^{-2}|\hat{u}|_{\mathcal{W}}^{2}+\nu^{-1} \lambda\right)^{\frac{1}{2}} .
$$

This number of controls is also sufficient if $L=M \hat{l}$, that is, if the partition of $\Omega$ is uniform: $\hat{l}_{i}=\hat{l}, i \in\{1,2, \ldots, M\}$.

In the general case we obtain, from Theorem 3.2, that it is enough to take a partition of $\mathcal{O}$ such that

$$
\hat{l} \leq \pi D_{1}^{\frac{1}{2}} \mathrm{e}^{-\frac{D_{2}}{2}\left(1+\left(\frac{\lambda}{\nu}\right)^{\frac{1}{2}}+\left(\frac{\lambda}{\nu}\right)^{\frac{2}{3}}+\frac{|\hat{u}|_{\mathcal{W}}}{\nu}+\frac{|\hat{u}|_{\mathcal{W}}^{2}}{\nu^{2}}\right)},
$$

which leads to the estimate on the number of needed controls

$$
M \geq \frac{l}{\pi} D_{1}^{-\frac{1}{2}} \mathrm{e}^{\frac{D_{2}}{2}\left(1+\left(\frac{\lambda}{\nu}\right)^{\frac{1}{2}}+\left(\frac{\lambda}{\nu}\right)^{\frac{2}{3}}+\frac{|\hat{u}|_{\mathcal{W}}}{\nu}+\frac{|\hat{u}|_{\mathcal{W}}^{2}}{\nu^{2}}\right)},
$$

where $l=l_{2}-l_{1}$ is the length of $\mathcal{O}$; this number is also sufficient if the partition is uniform.

\section{B. The gap between (16) and (18).}

The gap between (9) and (12) give rise to a big gap between (16) and (18). In Section VI, in order to understand if it is possible to improve (18), say that we also have an estimate like (16) in the general case, we consider the case of a uniform partition of $\mathcal{O}$, where (16) and (18) give also a sufficient number of controls. We present results of some numerical simulations comparing the number of controls

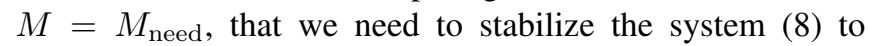
zero, with the following reference real numbers

$$
M_{\text {ref }}:=\frac{L}{\pi}\left(\nu^{-2}|\hat{u}|_{\mathcal{W}}^{2}+\nu^{-1} \lambda\right)^{\frac{1}{2}} ; \quad M_{\text {exp }}:=\frac{L}{\pi} \mathrm{e}^{M_{\text {ref }}}
$$

The value $M_{\text {ref }}$ is motivated by (16), and the value $M_{\text {exp }}$ by (18); notice that $\frac{l}{\pi} \mathrm{e}^{M_{\text {ref }}}$ is (roughly speaking, up to the constants $D_{1}$ and $D_{2}$ ) a lower bound for the right hand side of (18); we take $\frac{L}{\pi}$ instead of $\frac{l}{\pi}$ in front of $\mathrm{e}^{M_{\text {ref }}}$ in order to avoid giving the wrong idea that (18) goes to 0 with $l$ (indeed the constants $D_{1}$ and $D_{2}$ will increase as $l$ goes to 0 ).

In an uniform partition, we want to understand if a number of controls of the order of $M_{\text {ref }} \sim \frac{l}{\hat{l}}$ is also sufficient to stabilize the system (8) to zero, with rate $\frac{\lambda}{2}$.

\section{Feedback control and Riccati equation}

By the dynamic programming principle, and the arguments in [4, Section 3.2], we can derive the following result.

Theorem 4.1: The controls $\zeta$ given in Theorems 3.1 and 3.2 can be taken in feedback form

$$
\zeta=\mathrm{e}^{-\lambda t} P_{M} Q_{\hat{u}}^{t, \lambda} v
$$

for a suitable family of operators $Q_{\hat{u}}^{t, \lambda}: H \rightarrow H, t \geq 0$, with $\left|Q_{\hat{u}}^{t, \lambda}\right|_{\mathcal{L}(H \rightarrow H)} \leq \bar{C} \mathrm{e}^{\lambda t}$ for a suitable constant $\bar{C}$. Further, $Q:=Q_{\hat{u}}^{t, \lambda}$ satisfies the differential Riccati equation

$$
\dot{Q}-Q \mathbb{A}-\mathbb{A}^{*} Q-Q B_{M} B_{M}^{*} Q+\mathbb{L}=0
$$

where $\mathbb{L}=-\nu \mathrm{e}^{\lambda t} \partial_{x x}, \mathbb{A}=\mathbb{A}(t):=-\nu \partial_{x x}+\mathcal{B}(\hat{u}(t))$, $\mathcal{B}(\hat{u}) v:=\partial_{x}(\hat{u} v)$, and $B_{M}^{*}=B_{M}:=\mathrm{e}^{-\frac{\lambda}{2} t} P_{M}$.

\section{THE NONLINEAR OSEEN-BURGERS-LIKE SYSTEM.}

The next result is a corollary of Theorem 4.1. We omit the proof that follows by a stardard fixed point argument, following similar arguments as in [4, Section 4].

Theorem 5.1: Let $M$ be the integer in Theorem 4.1 (i.e., as in either (3) or (4)). Then there are positive constants $\Theta$ and $\epsilon=\epsilon(\Theta)$ depending only on $\lambda,|\hat{u}|_{\mathcal{W}}$, and $\nu$ such that for $\left|v_{0}\right|_{H} \leq \epsilon$ the solution $v$ of system (7), with $\zeta$ as in (20), is well defined for all $t \geq 0$ and satisfies the inequality

$$
|v(t)|_{H}^{2} \leq \Theta \mathrm{e}^{-\lambda t}\left|v_{0}\right|_{H}^{2} \quad \text { for } t \geq 0
$$

Notice that the feedback rule is found to globally stabilize to zero the linear Oseen-Burgers system (8). Then, Theorem 5.1 says that the same feedback rule also locally stabilizes to zero the bilinear system (7).

\section{NUMERICAL EXAMPLES: THE LINEAR SYSTEM}

We present some results of the numerical simulations we have performed concerning the stabilization of the OseenBurgers system (8) to zero. Below, $v_{\mathrm{u}}$ stands for the solution of the uncontrolled system (i.e., $\zeta=0$ ), and $v$ (or $v_{\lambda}$ ) stands for the solution of the system under the action of a feedback controller $\zeta=\mathrm{e}^{-\lambda t} P_{M} Q_{\hat{u}}^{t, \lambda} v$ as in (20). The rule $Q=Q_{\hat{u}}^{t, \lambda}$ is found by solving (21).

For the discretization we use finite elements in the space variable and a Crank-Nicolson scheme for the time variable. For further details we refer the reader to [11, Section 5].

We follow a "trying and checking" procedure, (i): we fix $M$, (ii): we (try to) compute the corresponding feedback control (the differential Riccati equation (21) has a solution only if $M$ is big enough), and (iii): we check the results of the simulations.

\section{A. A family of targeted nonstationary solutions}

We set $\nu=\frac{1}{10}, T=3, \Omega=(0, \pi), \mathcal{O}=\left(\frac{3}{2}, \frac{5}{2}\right), M=4$, $\lambda=4$, and the initial condition $v_{0}(x)=2 \sin (x)-3 \sin (2 x)$. Then we set the family of targeted solutions

$$
\hat{u}^{(i, j)}=C_{\mathrm{nr}}(\sin (-t) \sin (i x)-\cos (3 t) \sin (j x))
$$

where the constant $C_{\mathrm{nr}}$ is chosen such that $|\hat{u}|_{\mathcal{W}}=1$. Figure 1 shows, for different reference trajectories, that 
the system is not stable with rate $2=\frac{\lambda}{2}$, and that the feedback control is able to stabilize it with rate 2 . Notice that, accordingly Matlab, $M_{\text {ref }} \approx 11.83$ and $M_{\text {exp }} \approx 137610$.

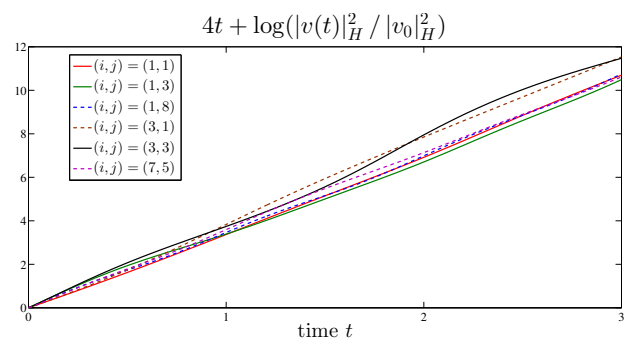

(a) Without feedback control.

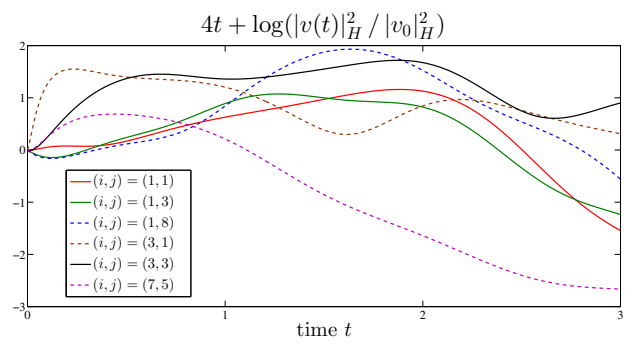

(b) With control.

Fig. 1. Stabilization for different reference solutions.

\section{B. An unstable situation}

Now we take $\nu=\frac{1}{10}, T=1, \Omega=\mathcal{O}=(0, \pi), M=4$, $\lambda=4, v_{0}(x)=\sin (x)$, and $\hat{u}=-\sin (5 x)$. In Figure 2 we see that the system is unstable. Figure 3 shows that one control is not enough to stabilize the system. We see that the optimal cost $(Q(t) v(t), v(t))_{H}=\int_{t}^{+\infty}(\mathbb{L} v, v)_{V^{\prime}, V}+$ $\left|B_{M}^{*} Q v\right|_{H}^{2} \mathrm{~d} \tau$, is not strictly decreasing, which contradicts the dynamic programming principle. This could explain the cuspy-like behavior of the norm of the control $\eta=B_{M}^{*} Q v$. In Figure 4 we see that two controls are enough to stabilize

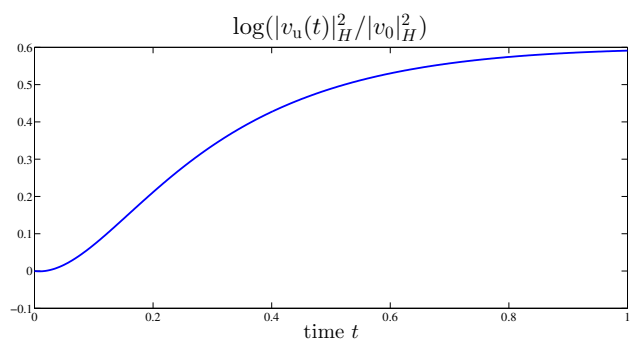

Fig. 2. Without feedback control.

the system with rate 2 . For $M=12>M_{\text {ref }} \geq \sqrt{140} \approx$ 11.83 we also observe that the behavior of the norms on time is more uniform. Finally the magnitude of the controls are shown in Figure 5, we see that with one control the magnitude is much bigger, which could rely on the fact that one control is not enough. For other simulations concerning this problem we refer to [11].

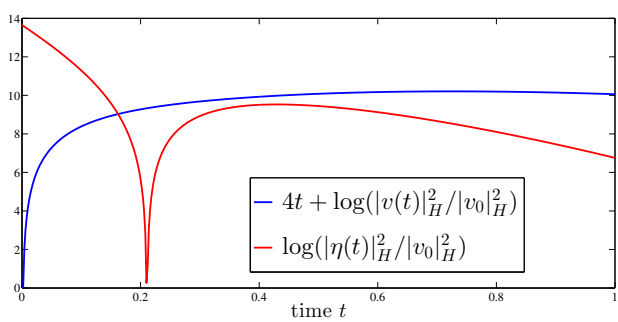

(a) The solution and control.

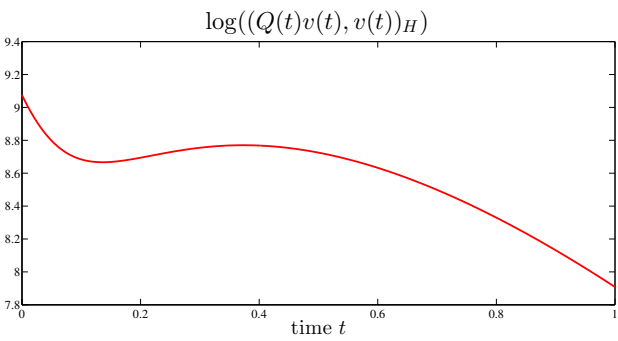

(b) The optimal cost.

Fig. 3. The case $M=1$.

\section{NumeriCAL EXAMPLES: THE BuRGERS SyStem}

It remains to confirm that the feedback control stabilizes system (1)-(2) to a given reference trajectory $\hat{u}$, provided $\left|u_{0}-\hat{u}(0)\right|_{H}^{2}$ is "small". We set the data as in Section VI-A, with $(i, j)=(3,3)$. Let $\delta \in \mathbb{R}$ and let $u$ solve (1)-(2) with $u_{0}=\hat{u}(0)+\delta v_{0}$ and with the feedback control $\zeta$, as in (20), computed to stabilize the linear system (8) to zero. We denote $d=u-\hat{u}$ and observe that, for $d_{0}:=d(0)=\delta v_{0}$, the local maxima of the function $\frac{\mathrm{e}^{\lambda t}|d(t)|_{H}^{2}}{\left|d_{0}\right|_{H}^{2}}$, in Figure 6(a), do not increase for $|\delta| \leq 0.1$, while they do for $|\delta| \geq 0.5$. That is, for small $|\delta|$ the feedback control is working and for big $|\delta|$ it is not.

\section{FINAL REMARKS}

We have presented a sufficient condition for stabilization to a nonstationary solution for the Burgers system by means of a finite dimensional controller. In the case of no constraint on the support of the control the condition leads to a much better estimate on the number of piecewise constant controls we need to stabilize the system; the results of numerical simulations we present suggest that a similar estimate should hold in the general constrained case.

\section{REFERENCES}

[1] M. Badra and T. Takahashi, "Stabilization of parabolic nonlinear systems with finite dimensional feedback or dynamical controllers: Application to the Navier-Stokes system," SIAM J. Control Optim., vol. 49, no. 2, pp. 420-463, 2011.

[2] V. Barbu, "Stabilization of Navier-Stokes equations by oblique boundary feedback controllers," SIAM J. Control Optim., vol. 50, no. 4, pp. 2288-2307, 2012.

[3] V. Barbu, I. Lasiecka, and R. Triggiani, "Abstract settings for tangential boundary stabilization of Navier-Stokes equations by high- and lowgain feedback controllers," Nonlinear Anal., vol. 64, pp. 2704-2746, 2006.

[4] V. Barbu, S. S. Rodrigues, and A. Shirikyan, "Internal exponential stabilization to a nonstationary solution for 3D Navier-Stokes equations," SIAM J. Control Optim., vol. 49, no. 4, pp. 1454-1478, 2011. 

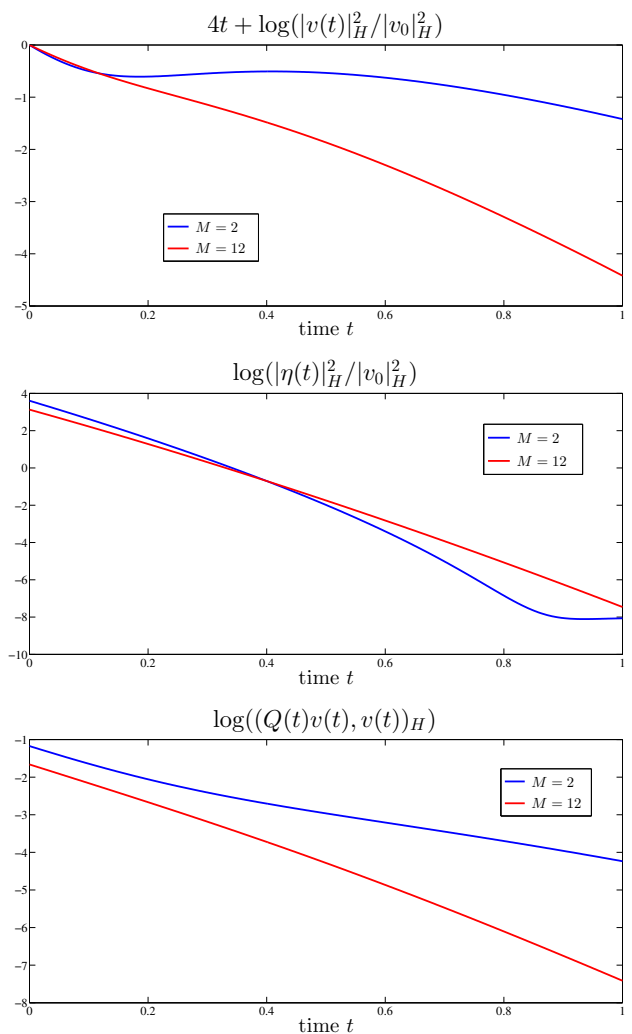

Fig. 4. The norms of solution and control, and the cost for $M \in\{2,12\}$.

[5] V. Barbu and R. Triggiani, "Internal stabilization of Navier-Stokes equations with finite-dimensional controllers," Indiana Univ. Math. J., vol. 53, no. 5, pp. 1443-1494, 2004.

[6] T. Duyckaerts, X. Zhang, and E. Zuazua, "On the optimality of the observability inequalities for parabolic and hyperbolic systems with potentials," Ann. Inst. H. Poincaré Anal. Non Linéaire, vol. 25, no. 1 , pp. 1-41, 2008.

[7] A. V. Fursikov and A. A. Kornev, "Feedback stabilization for the Navier-Stokes equations: Theory and calculations," in Mathematical Aspects of Fluid Mechanics, ser. London Mathematical Society Lecture Notes Series. Cambridge University Press, 2012, vol. 402, pp. 130-172 (ch. 7).

[8] M. Gunzburger, Perspectives in Flow Control and Optimization, ser. Advances in Design and Control. Philadelphia: SIAM, 2003.

[9] A. A. Kornev, "The method of asymptotic stabilization to a given trajectory based on a correction of the initial data," Comput. Math. Math. Phys., vol. 46, no. 1, pp. 34-48, 2006.

[10] — "A problem of asymptotic stabilization by the right-hand side," Russian J. Numer. Anal. Math. Modelling, vol. 23, no. 4, pp. 407-422, 2008.

[11] A. Kröner and S. S. Rodrigues, "Remarks on the internal exponential stabilization to a nonstationary solution for 1D Burgers equations," SIAM J. Control Optim. (to appear), 2015.

[12] M. Krstic, L. Magnis, and R. Vazquez, "Nonlinear control of the viscous Burgers equation: Trajectory generation, tracking, and observer design," J. Dyn. Syst. Meas. Control, vol. 131, no. 2, pp. 021 012(1-8), 2009.

[13] J.-P. Raymond and L. Thevenet, "Boundary feedback stabilization of the two-dimensional Navier-Stokes equations with finite-dimensional controllers," Discrete Contin. Dyn. Syst., vol. 27, no. 3, pp. 1159-1187, 2010

[14] S. S. Rodrigues, "Boundary observability inequalities for the 3D Oseen-Stokes system and applications," ESAIM Control Optim. Calc. Var. (to appear), 2014.
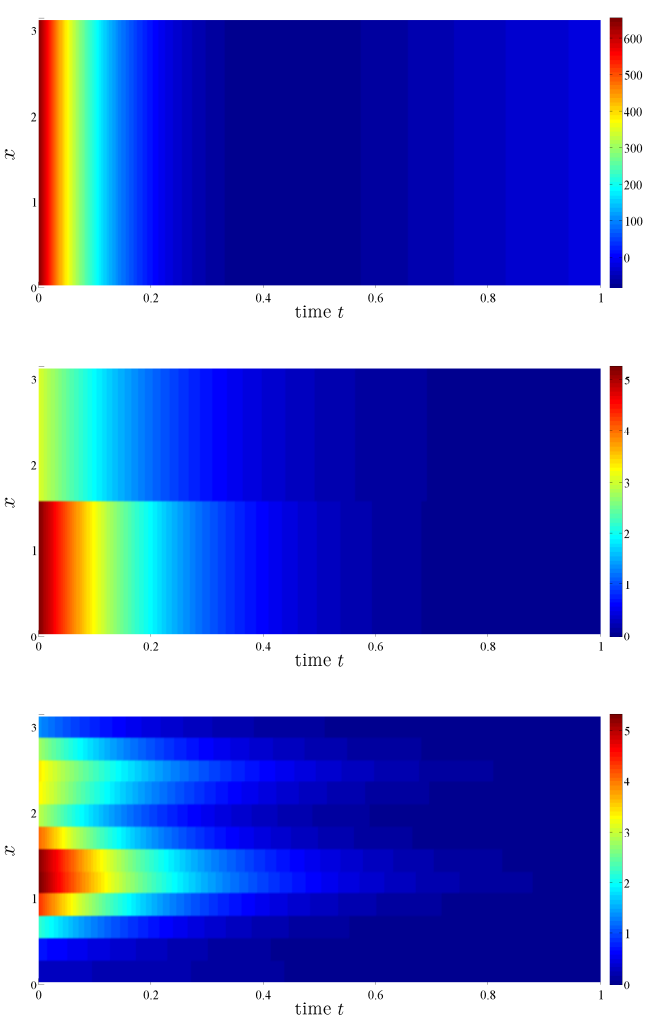

Fig. 5. The control for different $M \in\{1,2,12\}$.

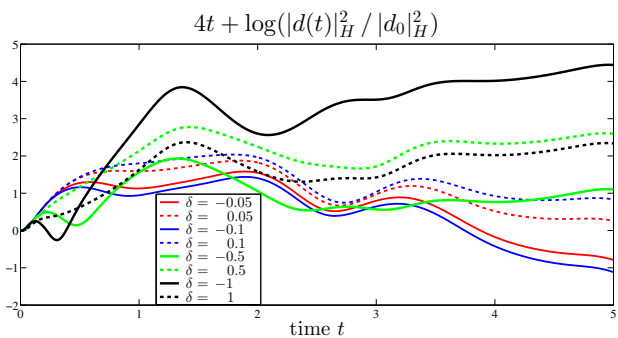

(a) The norm of the difference $u-\hat{u}$.

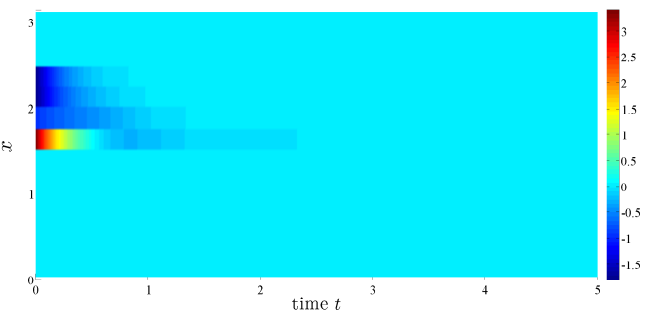

(b) The control for $\delta=-0.05$.

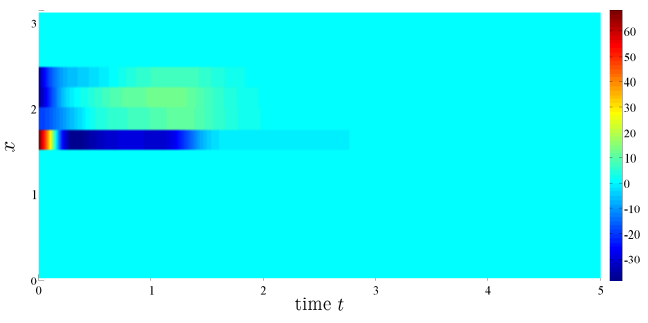

(c) The control for $\delta=-1$.

Fig. 6. Local stabilization for the Burgers system 